\title{
Educación corporativa y colaboración entre empresas y escuelas de negocios: Un estudio comparativo de casos
}

Corporate Education and Collaboration Dynamics Between Companies and Business Schools: a Comparative Case Study

\section{Volumen 22 Número 1}

Enero - Abril

pp. $1-28$

Roy Campos Retana

\section{Citar este documento según modelo APA}

Campos Retana, Roy. (2022). Educación corporativa y colaboración entre empresas y escuelas de negocios: Un estudio comparativo de casos. Revista Actualidades Investigativas en Educación, 22(1), 1-28. Doi. https://doi.org/10.15517/aie.v22i1.47435 


\title{
Educación corporativa y colaboración entre empresas y escuelas de negocios: Un estudio comparativo de casos \\ Corporate Education and Collaboration Dynamics Between Companies and Business Schools: a Comparative Case Study
}

Roy Campos Retana ${ }^{1}$

\begin{abstract}
Resumen: Aunque existe abundante literatura sobre la capacitación corporativa y estudios que la identifican como un ingreso importante para las escuelas de negocios se desconocen las dinámicas entre empresas y escuelas de negocios para desarrollar de manera exitosa los Programas de Capacitación a la Medida (PCM). El propósito de este artículo es explorar las dinámicas de colaboración entre empresas y escuelas de negocios para desarrollar con éxito los PCM de Latinoamérica. Debido a que son escasas las investigaciones previas, se desarrolló una investigación de carácter cualitativo y exploratorio en seis países de Latinoamérica: Argentina, Brasil, Colombia, Ecuador, México y Perú. Con base en la teoría fundamentada y mediante un estudio de casos llevado a cabo durante 2017 y 2018, se compararon cinco PCM desarrollados exitosamente con otros cuatro programas que presentaron dificultades. Se utilizó el programa ATLAS.ti, para el procesamiento y análisis de los datos. Los resultados permitieron responder a la pregunta de investigación ¿Cómo colaboran empresas y escuelas de negocios en el desarrollo de PCM de Latinoamérica? Los resultados sugieren que el éxito de los PCM dependen de dinámicas eficientes de colaboración entre ciertos agentes críticos de las empresas y de las escuelas de negocios a lo largo de tres fases: diagnóstico, diseño e implementación. Los hallazgos de esta investigación son novedosos porque amplían la literatura existente en capacitación corporativa, ayudan a comprender la colaboración en el campo de la educación ejecutiva, ofrecen criterios para optimizar la ejecución de los PCM y abren nuevas líneas de investigaciones futuras.
\end{abstract}

Palabras clave: relación industria y educación, educación superior, escuelas de administración de negocios, programas educativos de adultos.

\begin{abstract}
Although there is extant literature on corporate training and studies that identify it as a basic income for business schools, the dynamics between companies and business schools to successfully develop Customized Executive Programs (CEP) are unknown -by its acronym in Spanish "Programas de Capacitación a la Medida (PCM)"-. This article explores the dynamics of collaboration between companies and business schools to develop CEP in Latin America successfully. Since previous investigations are scarce, a qualitative and exploratory investigation was carried out in six Latin American countries: Argentina, Brazil, Colombia, Ecuador, Mexico, and Peru. Based on grounded theory and a case study carried out during 2017 and 2018, five CEP that was successfully developed is compared with another four CEP that presented difficulties. We used the ATLAS.ti software for data processing and analysis. The results allow us to answer the research question: How do companies and business schools collaborate in developing CEP in Latin America? The results suggest that the success of CEP depends on efficient collaboration dynamics between specific critical agents of companies and business schools throughout three phases: diagnosis, design, and implementation. The findings of this research are novel because they expand the existing literature on corporate training, help understand collaboration in executive education, offer criteria to optimize the execution of CEP, and open new lines of future research.
\end{abstract}

Keywords: corporate training, business schools, collaboration between business and academic sectors, adult education programs

${ }^{1}$ Profesor de la Escuela de Administración de Negocios, Universidad de Costa Rica, San José, Costa Rica. Master en Gobierno y Cultura de las Organizaciones, Universidad de Navarra, donde cursa sus estudios doctorales. Orcid https://orcid.org/0000-0001-7519-9219

Dirección eletrónica: roy.camposretana@ucr.ac.cr

Artículo recibido: 16 de junio, 2021

Enviado a corrección: 01 de octubre, 2021

Aprobado: 6 de diciembre, 2021

Los contenidos de este artículo están bajo una licencia Creative Commons 


\section{Introducción: Programas de Capacitación a la Medida en Latinoamérica}

Con el paso del tiempo, las organizaciones han adquirido mayor conciencia del potencial que tiene la educación de las personas que desempeñan cargos directivos (Büchel y Antunes, 2007) debido a que la enseñanza de la ciencia administrativa facilita que aprendan a desarrollar el liderazgo y a tomar mejores decisiones (Strebel, 2007). Esta tendencia ha llevado a que las escuelas de negocios colaboren cada vez más como aliadas de las organizaciones para el desarrollo de las destrezas directivas (Myrsiades, 2001). Como una consecuencia de estas tendencias, el diseño de los Programas de Capacitación a la Medida (PCM) ha pasado a tener un mayor nivel de colaboración entre las empresas y sus proveedores. Desafortunadamente, todavía hay un conocimiento bastante limitado sobre los criterios para estudiar los efectos de un PCM en la productividad de las personas que desempeñan cargos ejecutivos o de dirección (Asplund, 2004).

A pesar de esa relación creciente entre las empresas y las escuelas de negocios en el desarrollo de PCM, siguen siendo ambiguas las dinámicas de colaboración de estos programas (Lai, 2011). Las variables de éxito de un PCM no son las mismas para todas las empresas, puesto que los criterios de éxito varían de organización en organización, lo mismo que los sistemas de evaluación (Barnett y Mattox, 2010). Estos criterios cambian según la especialización del programa, la experiencia del personal de apoyo en una disciplina concreta y la orientación hacia clientes (Lockhart, 2013). Hay variables de colaboración que, independientemente de las culturas y de los sectores de la industria a los que pertenecen las empresas, siempre se presentan en los PCM para medir su éxito (Harrison y Shirom, 1999). Entre estas variables se encuentran: en la etapa de diagnóstico y diseño, formular las interrogantes apropiadas y tomar buenas decisiones (Murray, 2014); en la fase de implementación, las evaluaciones de satisfacción de las personas participantes (Kirkpatrick y Kirkpatrick, 2007); y posterior a la implementación, la medición del desempeño organizacional y el retorno sobre la inversión (Garavan et al., 2021; 2019).

Esta investigación responde a la pregunta ¿Cómo colaboran empresas y escuelas de negocios en el desarrollo de PCM de Latinoamérica? El propósito de este artículo es explorar las variables de colaboración de los PCM en América Latina, basados en un estudio comparativo de casos, diseñados e implementados por seis escuelas de negocios de la región. Este estudio exploratorio contribuye a la literatura de la administración mediante una aproximación a la colaboración y el aprendizaje entre organizaciones. De hecho, abre oportunidades de desarrollo para que las escuelas de negocios, adscritas a instituciones de 
educación superior, puedan organizar y gestionar de manera más eficiente su vinculación al sector productivo, así como fomentar la autonomía y la sostenibilidad de las unidades académicas que promueven estos formatos de transferencia del conocimiento. Para ello, se destacan los agentes claves que habilitan o restringen la colaboración exitosa de estos programas en tres áreas: diagnóstico, diseño e implementación.

\section{Referentes teóricos}

En términos generales, existen dos tipos de programas de formación ejecutiva: los programas abiertos o de libre inscripción, que son diseñados íntegramente por una escuela de negocios, y los PCM, que responden a las necesidades específicas de una organización y que son diseñados de manera conjunta entre la organización y una escuela de negocios (Montgomery, 2016). Mientras los programas abiertos son una solución ya preparada para atender ciertas lagunas de conocimiento de las personas que desempeñan cargos de dirección, los PCM los diseña una escuela de negocios en colaboración con los altos directivos de una organización. En los programas abiertos participan las personas que pagan una inscripción y cuentan con ciertos atributos del perfil de participantes, en cambio, en los PCM, participan únicamente personas escogidas estratégicamente por las personas encargadas de recursos humanos y capacitación de la organización (Dover et al., 2018).

Doh y Stumpf (2007) revelan que en los programas abiertos las personas que se inscriben suelen esperar un crecimiento personal y profesional, indistintamente del desempeño que tengan dentro de sus organizaciones; no obstante, en los PCM, las personas con cargos directivos tienen la expectativa de que la organización tenga un impacto positivo en su desempeño, en términos de cultura, desarrollo de destrezas, obtención de resultados, transformación organizacional, entre otros (Doh y Stumpf, 2007). Así, los PCM corren el riesgo de ser soluciones tan acotadas para una organización que pueden restringir el desarrollo de redes profesionales (networking) con individuos más allá de su empresa, industria y país. Pese a ello, en organizaciones muy grandes, pueden permitir desarrollar ese networking con colegas de otras divisiones, contextos culturales y diferente experiencia laboral, entre otras (Haskins et al., 2017).

En cuanto a la inversión económica, el costo de los PCM se convierte en una economía de escala en la medida en que se inscriben más participantes, porque diluye el costo total entre mayor cantidad de personas inscritas y, por tanto, el precio por asistente se reduce considerablemente e, incluso, puede ser menor que el de un programa abierto (Carrigtoncrisp, 
2017). Vista en su conjunto, la educación de personas que desempeñan cargos directivos funciona como un catalizador de las organizaciones, y los PCM juegan un rol importante en la transformación de sus culturas, así como en las dinámicas de colaboración dentro de ellas y entre ellas (Kets De Vries y Korotov, 2007).

\subsection{Colaboración y aprendizaje}

La colaboración es "una relación mutuamente beneficiosa y bien definida, introducida por dos o más organizaciones para lograr metas comunes" (Mattessich y Johnson, 2018). Para el desarrollo de los PCM, la colaboración entre una empresa y una escuela de negocios implica numerosas interacciones orientadas a la resolución de una problemática propia del sector o de la empresa que puede solventarse, total o parcialmente, mediante educación de personas con cargos directivos. Para tal fin, las personas que participan en el diseño e implementación de un PCM procuran mantener una visión compartida y, como fruto de la colaboración entre ellas, surgen numerosas actividades sincronizadas y coordinadas (Leach et al., 2014). No obstante, esas colaboraciones mutuas son difíciles, precisamente por la pluralidad de intereses que convergen entre las organizaciones para lograr metas comunes de forma colaborativa (Kwan, 2019).

La colaboración necesita sistemas de aprendizaje organizativo con tal de hacer sostenibles las relaciones institucionales, optimizar los procesos y alcanzar los resultados acordados (Doz, 2007). La colaboración surge en la medida que se procuran crear marcos de referencia similares para comunicar y transferir nuevos conocimientos de manera eficiente (Knoben y Oerlemans, 2006). La generación de un producto o servicio es factible gracias a una relación favorable entre dos o más organizaciones (en ocasiones suele haber presencia de un tercer actor; por ejemplo, otro proveedor) que buscan una meta común (Goossen, 2015). En consecuencia, la colaboración de un PCM no se mide únicamente por la creación de un nuevo producto o servicio, sino por la eficiencia de las dinámicas entre las personas y sus organizaciones (Gulati et al., 2012).

Considerando que el aprendizaje organizacional es una modificación del desempeño organizacional como resultado de la experiencia (Madsen y Desai, 2010), la interacción entre las personas de la empresa y la escuela de negocios conlleva un proceso de aprendizaje organizacional que, gestionado apropiadamente, permite resolver una necesidad organizacional. Por ejemplo, la transferencia del conocimiento desde el aula hasta el entorno de trabajo es, en gran medida, posible, gracias al reto que asumen las personas docentes para 
generar nuevos aprendizajes en los participantes. En consecuencia, el aprendizaje organizacional es importante en el desarrollo de los PCM, en la medida que exige compromisos recíprocos de los individuos entre sí, de acuerdo con el espíritu de la organización (Senge, 1990). Por tanto, resulta necesario analizar aún más las dinámicas de colaboración y aprendizaje -individual y organizacional- para comprender los comportamientos que habilitan el éxito de los PCM.

\section{Metodología}

Como se explicó antes, la investigación previa sobre las dinámicas de colaboración en los PCM y sus factores de éxito y fracaso es muy escasa, por lo que se exploró el fenómeno por medio de un estudio comparativo de casos. El estudio comparativo de casos involucra el análisis y síntesis de las similitudes, diferencias y patrones en dos o más casos que comparten un enfoque u objetivo común (Yin, 2014). Esta metodología de investigación facilitó generalizar cuestiones causales para entender las divergencias y convergencias entre las empresas y las escuelas de negocios en las dinámicas de co-creación de un PCM.

El estudio comparativo de casos es preferible en tres condiciones (Pera et al., 2016): primero, cuando se plantean las preguntas "cómo" o "por qué"; segundo, cuando el investigador tiene poco control sobre los acontecimientos; y, tercero, cuando se centra en un fenómeno contemporáneo dentro del contexto de la vida real. Para analizar cómo fluye la colaboración en el desarrollo de los PCM, se efectuó un estudio comparativo de casos, llevado a cabo durante 2017 y 2018, el cual permitió desarrollar un examen detallado de un fenómeno de la vida real (Eisenhardt, 1989). En concreto, se analizaron las dinámicas de colaboración para el desarrollo de los PCM, con variedad de países, culturas y sectores empresariales.

\subsection{Unidades de análisis}

Se siguieron de cerca los procesos propuestos por Yin (2014), para quien un caso como unidad de análisis puede ser un programa, una institución, una persona, un proceso o una unidad social. Para ello se seleccionaron nueve PCM, desarrollados en seis países de Latinoamérica: Argentina, Brasil, Colombia, Ecuador, México y Perú. La selección de los PCM fue por conveniencia debido a que pertenecían a una red de escuelas de negocios asociadas. La intención al elegir PCM de varias nacionalidades, extensión y tipo de industria fue la de contrastar los hallazgos en múltiples casos que, en ciertas situaciones, resultaron sumamente fructíferos (Merriam, 1998). Por consiguiente, se requirió, al mismo tiempo, de diversidad y 
similitud, de manera que contaran con factores comunes y elementos distintivos para compararlos entre sí. Los PCM estudiados se habían ejecutado recientemente o, incluso, estaban siendo implementados en el momento de aplicar las entrevistas. Esto nos permitió contar con insumos más recientes, y que las personas informantes recordaran la experiencia del PCM fácilmente, lo cual fue de especial relevancia, pues eran programas con un alto nivel de personalización (Yin, 2008). Los PCM estaban dirigidos, en su mayoría, a personas en posiciones directivas de las empresas o que aspiraban a desempeñarse en roles ejecutivos, provenientes de empresas nacionales, internacionales y multinacionales.

Para efectos de este estudio, se identificaron como PCM exitosos aquellos que superaron oportunamente las dificultades que se presentaron a lo largo de su desarrollo, y los PCM de fracaso, como los que no gestionaron bien las dificultades a lo largo de las etapas de diagnóstico, diseño e implementación. Las seis escuelas de negocios participantes formaban parte de una red de instituciones asociadas, por lo que el acceso a los contactos fue más ágil, así como su transparencia y apertura. Todas estas escuelas tenían la particularidad de utilizar el método del caso como estrategia didáctica principal, por su probada efectividad en el aprendizaje de profesionales (Anderson et al., 2014). Se aplicaron al menos seis entrevistas semi estructuradas por cada caso de estudio, para un total de 62 entrevistas agrupadas en nueve casos de estudio. Por cada PCM, se procuró que la mitad de los informantes representaran a la escuela de negocios y la otra mitad representara a la empresa (ver el anexo 1 para la distribución y perfil de las empresas estudiadas).

\subsection{Técnicas de recolección}

La recopilación de datos se basó, totalmente, en entrevistas semiestructuradas hasta completar los nueve casos de estudio de carácter exploratorio (Boyce y Neale, 2006). Las entrevistas en profundidad son útiles cuando se desea obtener información detallada sobre las creencias y comportamientos de una persona, o si se desea explorar nuevos temas a fondo (Yin, 2014). Primero, hubo un acercamiento a las personas encargadas de los PCM por correo electrónico y se invitó a seis escuelas de negocios a participar en el estudio; en ocasiones fue necesaria la autorización de la dirección de la escuela y de cartas de presentación para explicar la investigación. Aunque se ofreció firmar un consentimiento informado para proteger la identidad de las instituciones y personas participantes, en ningún caso fue necesario firmar acuerdos formales de confidencialidad. Luego de aprobar la participación de la escuela de negocios, facilitaron los contactos de las personas que tenían el perfil requerido, los cuales se 
abordaron por correo electrónico, con el apoyo del departamento de PCM o, incluso, de manera autónoma.

Se utilizaron estudios sobre los PCM para desarrollar una matriz de áreas a abordar en tres etapas del desarrollo de cada PCM (Asplund, 2004; Doh y Stumpf, 2007; Lockhart, 2013): antes, durante y después de la implementación del PCM (ver el anexo 2 para el la guía de la entrevista). El tiempo de cada entrevista osciló entre 20 y 89 minutos, y la duración promedio fue de 45 minutos. En total, se efectuaron 62 entrevistas semi estructuradas para una duración acumulada de 46.93 horas. Las entrevistas se realizaron vía Skype, y se grabó el audio con el consentimiento previo de las personas informantes, para facilitar el posterior análisis de la información (Yin, 2009). A lo largo del trabajo de campo, se recabó alguna información adicional de primera mano: cronogramas, contenidos, metodología, entre otros.

\subsection{Procesamiento de análisis}

El análisis siguió técnicas establecidas y procedimientos para la construcción de la teoría fundamentada (Glaser y Strauss, 1999). El estudio de los datos comenzó con un análisis de las entrevistas y fue seguido por una comparación de casos en un proceso de codificación de dos ciclos. Se empleó Atlas.ti 8.3.0 para iOS. En el primer ciclo, se codificaron las entrevistas de manera independiente, por grupos de casos, examinando los patrones relacionados con el desarrollo de los PCM y las variables que influyeron en el diseño e implementación. Este proceso generó una lista de códigos relacionados con las estrategias utilizadas para la gestión de los PCM. En este primer ciclo del análisis, las transcripciones de las entrevistas, que fueron ingresadas como archivos de texto en Atlas.ti, se analizaron sobre la base de códigos previamente determinados en la matriz de variables, así como sobre palabras "in vivo".

A partir de esas 62 entrevistas analizadas, se emitieron reportes de Atlast.ti con las principales citas relacionadas a esos códigos, agrupadas según los nueve PCM, y desde allí se redactaron los respectivos casos de estudio. Las familias de documentos a los cuales se aplicaron los filtros por códigos permitieron seguir un cierto orden en la redacción de casos, de manera que posteriormente facilitó su orden y estructura. Los casos de estudio fueron enviados a los principales "sponsors" de cada escuela de negocios para que los revisaran y verificaran que la historia del caso correspondía plenamente a lo sucedido en la realidad. En total, se escribieron 504 páginas correspondientes a nueve casos de estudio (ver el anexo 3 para información adicional sobre la fundamentación de variables). 
En el segundo ciclo de codificación, se realizó un análisis sistemático de casos en el que se compararon y contrastaron los hallazgos (Saldana, 2016). En este segundo ciclo de análisis, se codificaron los casos de estudio de manera independiente, para generar códigos de mayor nivel de abstracción para lograr una codificación axial hasta crear un total de 41 códigos. Luego, se generaron grupos de redes para identificar las relaciones entre las distintas categorías, los códigos y sus definiciones. Esto permitió revisar las reiteraciones conceptuales y subsumir los códigos que causaban algún tipo de repetición en otros más pertinentes (ver el anexo 4 para una vista parcial del libro de códigos). Finalmente, se generaron tablas de frecuencia y gráficas para conocer la fundamentación de los códigos a lo largo de los diferentes casos de estudio. Estas múltiples iteraciones permitieron encontrar las dinámicas que explican la colaboración en el desarrollo de los PCM. En este documento no se evidencian tablas de frecuencia ni gráficas.

\section{Resultados y discusión}

Los datos analizados desvelaron una serie de comportamientos que vincularon la colaboración de los PCM con varios agentes que interactuaron entre sí, principalmente entre la Dirección del PCM de la escuela de negocios y los encargados de Aprendizaje y Desarrollo (AyD) de la empresa. Se identificaron que estos agentes habilitaron o restringieron la colaboración en tres etapas de su desarrollo: diagnóstico, diseño e implementación de los PCM.

En todos los PCM estudiados, se dio un impulso inicial por parte de alguna autoridad de la empresa para la contratación de un PCM con una escuela de negocios. Al inicio hubo actividades separadas, donde la empresa y la escuela de negocios funcionaron de manera aislada, pero posteriormente se dio una mayor cantidad de interacciones, originando puntos de convergencia. Las dinámicas de colaboración propias de un PCM no estaban sujetas a reglas fijas, como se verá más adelante.

Por parte de la escuela de negocios, intervinieron especialmente tres agentes. En primer lugar, la Dirección del PCM: era la persona responsable de la comercialización y gestión de propuestas de formación a la medida. Por un lado, recibía las solicitudes del cliente y, por otro, gestionaba los recursos internos de la escuela de negocios -logística, administrativos, contenidos, profesorado, entre otros-. En segundo lugar, la Dirección Académica: era la persona co-responsable de los contenidos del PCM, el equipo docente y los materiales, de acuerdo con las necesidades expresadas por cliente. En tercer lugar, la Dirección del 
Programa, la persona responsable del acompañamiento del grupo durante la implementación del PCM.

Por parte de la empresa, intervino principalmente el departamento de AyD, que usualmente dependía del área de Recursos Humanos. El departamento de AyD solía estar liderado por un profesional que, por iniciativa de un área funcional o jerarquía de la organización, identificaba necesidades internas de la empresa susceptibles de ser solventadas mediante formación a la medida. Así, la persona encargada de AyD era el responsable de la convocatoria y selección de proveedores, así como el diseño e implementación de los programas de capacitación. En negociaciones más avanzadas le correspondía gestionar reuniones con personas internas a la empresa -ej., directivos, gerentes-y con la Dirección del PCM de la escuela de negocios.

Cada uno de los agentes mencionados anteriormente -en la empresa y la escuela de negocios- tenían puntos de vista distintos, propios de las actividades de su organización, pero, al mismo tiempo, dominaban ciertos conocimientos comunes, propios del ámbito de la capacitación corporativa. En ocasiones, los distintos agentes asignaban nombres diferentes al PCM: programa in-company, workshop, programa de liderazgo. Esto se hacía más evidente en las reuniones de cliente-proveedor. Las direcciones de cada entidad tenían, a su vez, áreas de soporte que, posteriormente, colaboraban en el diagnóstico, diseño e implementación del PCM. Por ejemplo, los profesionales de AyD abordaban a las personas encargadas de cada área de negocios de la empresa: gerencia, operaciones, finanzas, entre otros; y la Dirección del PCM y la Dirección Académica, abordaban a quienes coordinaban cada área académica (finanzas, mercadeo, entre otros) para que estos, a su vez, contactaran al personal docente idóneo e identificaran los materiales más pertinentes a los objetivos de aprendizaje especificados. En la Figura 1 se observa mejor cómo se coordinaron las actividades locales de colaboración, entre agentes de la escuela de negocios y de la empresa. 
Figura 1

Dinámicas de colaboración entre agentes de la escuela de negocios y de la empresa

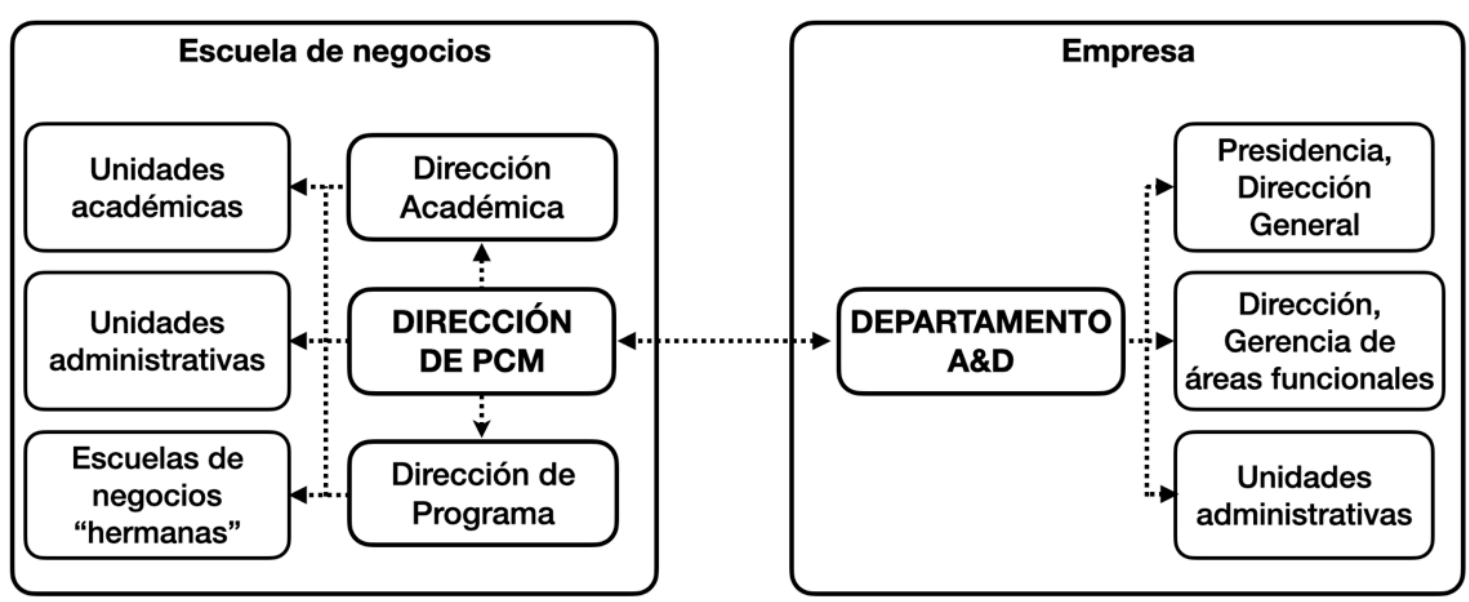

Fuente: Elaboración propia, 2021

En algunas ocasiones, la Dirección del PCM convocaba directamente a las personas docentes para el diseño y solo en algunas ocasiones pedía soporte -al profesorado, a la Dirección Académica, o a quienes coordinaban el área académica- para participar en reuniones con la persona encargada de AyD. Esto último sucedía en situaciones donde el PCM tenía cierto nivel de complejidad o cuando las negociaciones del PCM estaban ya avanzadas. Luego, se enviaba una propuesta preliminar del PCM con contenidos y precios. Si existía una aceptación inicial por parte de la empresa, entonces se procedía a elaborar los contenidos de una manera más detallada. Esta dinámica interna de la escuela de negocios era similar en todos los PCM.

Todas las interacciones que se presentaron entre los agentes a lo largo de este flujo eran dinámicas de colaboración. En general, los departamentos de PCM tenían la responsabilidad de hacer un diagnóstico de la necesidad de formación de la empresa, y si la empresa ofrecía un diagnóstico debían validarlo, o bien, realizarlo de manera conjunta En algunos PCM variaba la dinámica o la nomenclatura de los cargos, pero las tareas eran esencialmente las mismas. Por ejemplo, la Directora del PCM \#8 trabajaba como Directora del Programa y Académica: desempeñaba funciones de diseño en el PCM -contactar docentes, escogencia de materiales didácticos-, a la vez que estaba presente en todas las sesiones del PCM (ver Figura 2). 
Figura 2

Dinámicas de la colaboración en el diagnóstico, diseño e implementación

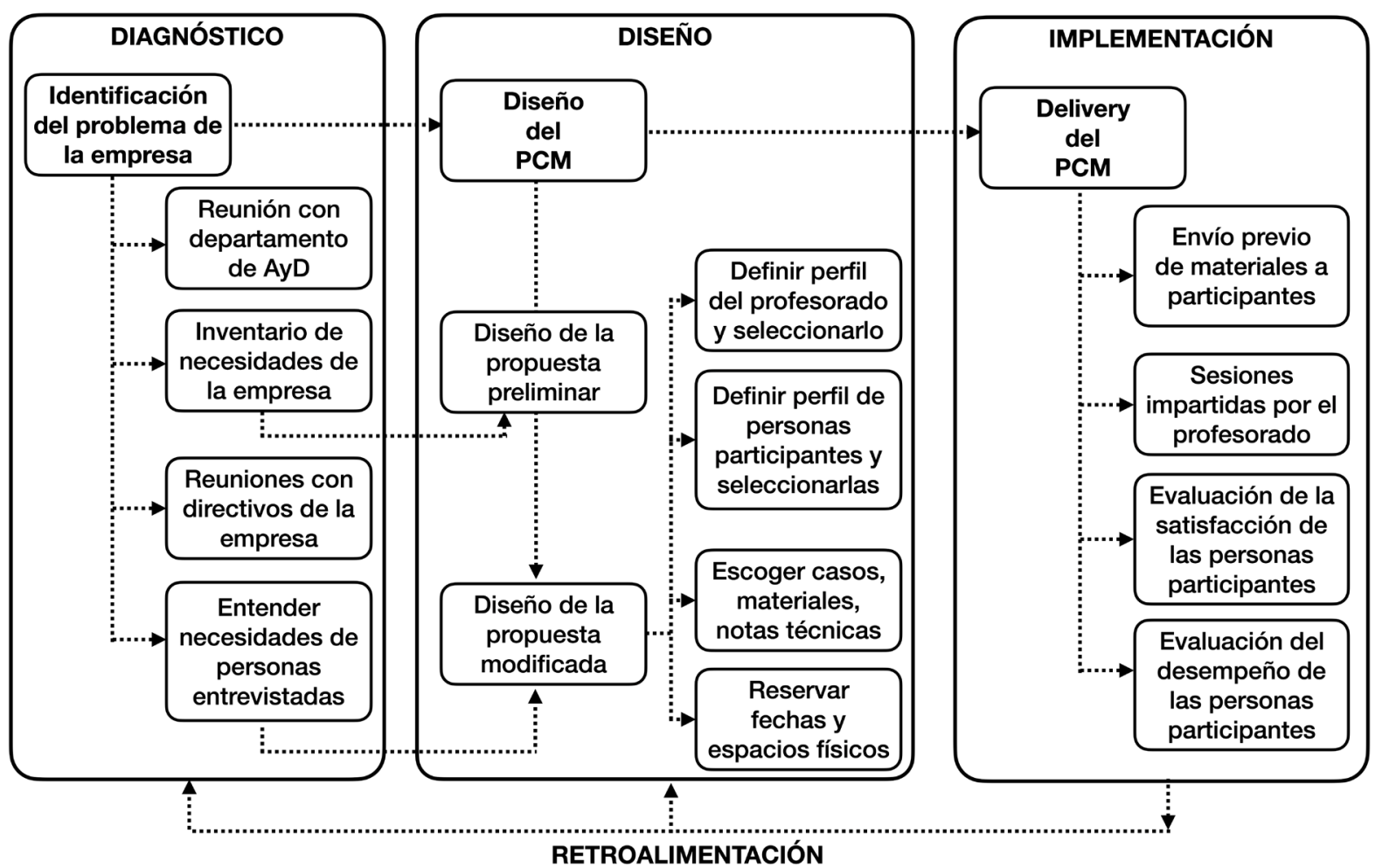

Fuente: Elaboración propia, 2021

Ahora se desarrollará la explicación de cada una de esas dinámicas de colaboración: diagnóstico, diseño e implementación.

\subsection{Diagnóstico}

Uno de los factores críticos en las dinámicas de colaboración era el diagnóstico de las necesidades de la organización para identificar sus requerimientos de formación. Por parte de la empresa se requería que la persona encargada de AyD tuviera claridad en sus necesidades de formación. Por ejemplo, en los PCM de éxito se logró identificar la necesidad y su causa; en cambio, en los PCM con dificultades era frecuente que se pidiera a la escuela de negocios la solución que considerara oportuna, sin expresar su necesidad, ni hacer un diagnóstico conjunto de ella. Las buenas propuestas de PCM eran posibles cuando existía un alto nivel de transparencia por parte de la persona encargada de $\mathrm{AyD}$, y una especial pericia por parte de la Dirección de PCM en el momento de entrevistarse con informantes clave de la empresa. 
Por parte de la Dirección del PCM, un buen diseño exigía entender qué quería la empresa, al tiempo que debía coordinar los recursos internos de la escuela de negocios para diseñar el PCM. En los PCM exitosos, las dinámicas de colaboración durante la fase de diagnóstico permitieron hacer explícitos los problemas de la empresa a fin de tomar decisiones de capacitación pertinentes. Las decisiones conjuntas ayudaron a que las sucesivas ediciones de un PCM fueran mejores que las anteriores. Una comunicación fluida provocó que pudiera emerger un mayor conocimiento entre las partes logrando así que la colaboración se consolidara. Por ejemplo, el PCM \#3 estaba dividido en dos partes, y el nivel de satisfacción de la segunda parte fue superior al de la primera, porque hubo un mayor conocimiento entre las organizaciones y las necesidades de formación.

En los PCM menos exitosos, la falta de comunicación entre ambas organizaciones restringió el nivel de colaboración hasta provocar dificultades en el diagnóstico del PCM. Por ejemplo, algunos departamentos de AyD confundieron los temas del PCM con los contenidos del PCM, y en lugar de apuntar a objetivos de aprendizaje, se enfocaban en nombres o actividades de enseñanza. No obstante, no todos los diagnósticos permitieron implementar exitosamente un PCM. Por ejemplo, en el PCM \#7 se hizo un diagnóstico conjunto y pertinente, pero la implementación del PCM presentó muchas dificultades, las cuales obedecieron principalmente a la heterogeneidad del grupo, como se verá más adelante.

Tanto las direcciones de los PCM como su equipo de soporte eran personas con importante trayectoria en el campo de la capacitación corporativa, específicamente en la comercialización y el desarrollo de proyectos formativos a la medida. Su perfil era más de gestión que académico, pero lograban equilibrar ambas facetas para articular las necesidades de los clientes con las posibilidades de la escuela de negocios: eran capaces de hacer un match entre ambas organizaciones. Algo similar sucedió con las personas que estaban a cargo de los procesos de AyD: poseían trayectorias importantes en su campo, concretamente en la capacitación y gestión del talento, inclusive, ya habían diseñado PCM anteriormente. Lo contrario desencadenó dificultades en los PCM. Por ejemplo, en el PCM \#9 la dirección de la empresa no tenía experiencia en contrataciones de capacitación, ni una Dirección de AyD; luego, contrató un PCM de alta dirección para un perfil de mandos medios, el cual obtuvo resultados regulares.

Los PCM con dificultades presentan errores en la identificación de las necesidades de la empresa, provocados por quienes intervinieron, tanto de la empresa como de la escuela de negocios. Tal fue el caso del PCM \#6, donde se dio un proceso de subestimación recíproco 
entre ambas partes. Por una parte, la empresa cuestionó el diagnóstico de la escuela de negocios, los contenidos y docentes que se asignaron al PCM; no así la metodología que, de antemano, se sabía que era el método del caso. Por otra parte, la escuela de negocios cuestionó si el diagnóstico de AyD era el apropiado; por ejemplo, que el problema no era de liderazgo, sino de comunicación. En consecuencia, el diagnóstico fue impreciso: la desconfianza sesgó la definición del problema, por el escepticismo de una o de ambas partes.

Cada interacción entre individuos de la empresa y la escuela de negocios requirió establecer un lenguaje común, el cual exigió un esfuerzo en ambos sentidos. Cuando la persona encargada de AyD hacía una solicitud tenía una intención detrás de su petición; al mismo tiempo, cuando la Dirección de PCM recibía la petición debía reflexionar sobre lo que realmente necesitaba la empresa, de manera que pudiera gestionar con independencia aquellos aspectos explícitos y tácitos de sus necesidades. Este era un caso patente de colaboración que, a su vez, evidenciaba el perfil experto de las direcciones de PCM: se trataba de profesionales con maestría en su campo, con destrezas para trabajar con personas de otros sectores empresariales y tipos de organizaciones.

\subsection{Diseño del PCM}

El diseño del PCM fue uno de los factores críticos en las dinámicas de colaboración. En los PCM de éxito hubo continuidad de las personas que intervinieron en el diseño y, si cambiaban, eran sustituidas por otras con experiencia en el diseño de PCM. Esto reflejaba que la colaboración era un comportamiento procurado, el cual era absorbido por la cultura de las organizaciones; distinto a los PCM de fracaso, donde el lenguaje común estaba asociado a personas particulares, no por la cultura de la organizacional. El perfil de las direcciones de PCM era, en la mayoría de las ocasiones, el apropiado. No obstante, en los PCM con dificultades, las personas a cargo de los procesos de AyD no tenían un perfil tan experto: por algún motivo desconocían el oficio del diseño de programas a la medida y esto provocaba sucesivas complicaciones del PCM.

La capitalización de la experiencia adquirida en los procesos de diseño de los PCM era un factor clave que se repitió en los diferentes contextos de este estudio: ese conocimiento se concentró en las personas que coordinaban los PCM. Por ejemplo, en el PCM \#7, la discontinuidad de profesionales de la institución que contrató el PCM impidió acumular el aprendizaje; esto se explica por la temporalidad de la colaboración, y al mismo tiempo, porque el lenguaje común desarrollado -particularidades de la capacitación corporativa- no se arraigó 
en la subcultura de la organización, lo que causó nuevas divergencias. En cambio, en el PCM \#5, al igual que en otros PCM, hubo recompras y consolidación de las relaciones a lo largo del tiempo, lo cual facilitó el rediseño del PCM -ajustes y actualizaciones-, e incrementó el conocimiento entre las partes.

Parte importante de las actividades de diseño estribaba en identificar los perfiles de los profesionales que participarían en el PCM y buscar una apropiada composición de grupos. De hecho, además de la interacción entre la Dirección de PCM y los encargados AyD, había otros dos perfiles relevantes: el perfil de docentes y participantes. El perfil del profesorado presentaba tres características: primero, su formación académica -ej., doctores-; segundo, su trayectoria empresarial -ej., individuos en cargos directivos-; tercero, sus destrezas como docentes en grupos de profesionales con experiencia directiva. En las entrevistas se definió su perfil como "holístico" y, en general, todos tenían algún tipo de trayectoria empresarial o formación internacional en instituciones de prestigio.

Escoger docentes con un perfil más académico o empresarial variaba en cada PCM. Por ejemplo, si se necesitaban docentes con experiencia profesional concreta -ej., sector bancario-, o si en un programa era necesario complementar la cantidad de docentes de perfil académico con otros de un perfil más empresarial. En ocasiones, para lograr este balance y complementar el claustro de docentes locales, se acudía a escuelas de negocios "hermanas", y se solicitaba el apoyo de docentes internacionales. No se registraron contrataciones de consultores independientes.

La trayectoria de los participantes de los PCM presentaba rasgos profesionales que luego tenían un efecto positivo o negativo en la constitución del grupo del CEP. En cuanto al perfil profesional, en su mayoría, dominaban la gestión de su negocio, ocupaban posiciones estratégicas y poseían potencial de crecimiento. Tenían responsabilidades importantes -ej., administrativas, de personal-, en ocasiones en varios países. Necesitaban prepararse para transformaciones organizacionales o cambios del sector industrial de su empresa mediante el desarrollo de destrezas directivas -ej., creatividad, resolución de problemas-. En los PCM exitosos, la mayoría de los participantes habían trabajado durante varios años en la organización hasta asumir posiciones gerenciales, o contaban con poca trayectoria en la empresa, pero con mucho potencial de crecimiento en la organización. Lo anterior habilitaba la homogeneidad de los grupos del PCM.

La homogeneidad de un PCM era la composición de los participantes de un grupo a través de una actividad de "curación" en aspectos tales como la edad, profesión, potencial de 
desarrollo y actitud hacia el aprendizaje, para lograr una composición diversa y multidisciplinaria. Mientras los grupos heterogéneos restringían las interacciones de las personas participantes, los grupos homogéneos habilitaban el aprendizaje, elevando así la calidad del PCM y su satisfacción. Esta labor de "curación" la realizaban entre las personas encargadas de AyD y la Dirección de PCM. En los casos exitosos, las dinámicas de colaboración eran más efectivas: no solamente definían un perfil de participante, sino que incluso los seleccionaban nominalmente. De hecho, los PCM exitosos se caracterizaban por un alto nivel de exigencia en la selección de los participantes.

En cambio, en los PCM que experimentaban dificultades, las personas participantes tenían un perfil muy heterogéneo, lo cual entorpecía la colaboración en el proceso de comunicación y aprendizaje. Tal fue el PCM \#7, un programa de juntas directivas, al que asistieron amas de casa y empresarios; y el PCM \#9, un programa de habilidades directivas, al que asistieron jefaturas y coordinaciones de área, pero nunca directivos. Las direcciones de estos PCM lo hicieron notar a sus interlocutores en las empresas, quienes hicieron caso omiso a esta advertencia. En consecuencia, se afectó el desempeño del PCM y sus niveles de satisfacción, ya que se ofreció una solución directiva a un grupo de asistentes que ocupaban direcciones intermedias en sus organizaciones, o incluso un perfil más operativo.

Las direcciones de PCM, además de diseñar un producto nuevo con profesionales de la empresa -clientes externos-, también lo diseñaban con docentes de la misma escuela de negocios -clientes internos-. Los PCM con mayor éxito eran aquellos donde la Dirección del PCM lograba un match apropiado entre la realidad de la empresa y la realidad que se presentaba con los casos, o con la temática impartida por el profesorado. En ocasiones, las direcciones de PCM transmitían la necesidad y objetivos del PCM a los profesores: sabían encontrar analogías entre la realidad de los casos y la realidad de la empresa. En general, se percibía que los docentes de los PCM exitosos diseñaban sesiones de calidad con mucho detalle. Parte del éxito de esas sesiones estribaba en que los profesores tenían la capacidad de "romper esquemas mentales" -ej., paradigmas- de las personas participantes, en gran medida por la conexión generada entre ambos contextos durante la implementación de sus sesiones con el método del caso.

\subsection{Implementación}

Las dinámicas de colaboración también fueron críticas en la fase de implementación de los PCM. El aprendizaje demandó una actitud de colaboración en ambas partes -profesores y 
aprendices-, para que fuera factible la fluidez en la transmisión de los contenidos. Dentro de los PCM exitosos, los participantes con un mejor desempeño eran los interesados en su desarrollo profesional y, al mismo tiempo, quienes habilitaron una apropiada implementación: tenían expectativas altas sobre el PCM y mantenían un mayor compromiso -ej., lectura, estudio, participación-. Esto permitió llenar la expectativa de las empresas con respecto al PCM, y que la capacitación tuviera incidencia en el desempeño profesional. Por ejemplo, hubo empresas que, como efecto del PCM, confirmaron la reducción en las tasas de rotación de personal, el incremento de las ventas o mejoras en el clima laboral.

Con respecto a las habilidades docentes, el profesorado de los PCM dirigía sus clases con estrategias didácticas de aprendizaje activo, principalmente el método del caso. En general, poseían un conjunto de casos que habían utilizado exitosamente en ocasiones anteriores hasta el punto de dominarlos con especial destreza. El uso de materiales nuevos en una sesión de directivos con experiencia tenía resultados variables: en el PCM \#3 el resultado fue favorable, porque la persona docente estudió los estados financieros de la empresa y generó una dinámica colaborativa de "workshop" para aportar soluciones concretas.

En la mayoría de los PCM estudiados, el profesorado utilizó casos distintos al contexto o sector de la empresa y eso no significó un problema, ni siquiera en los PCM con dificultades. El balance en un mismo docente era excepcional -académico vs empresario-, por eso se buscaba el balance en la "curación" del profesorado de todo el PCM en su conjunto. La buena relación del profesorado con las personas participantes ayudaba a convertir el salón de clases en un área de colaboración, donde se desarrollaron discusiones propias del método del caso para que se intercambiara el conocimiento abstracto de la disciplina particular de los negocios con el conocimiento específico del caso.

En los PCM exitosos, los profesores aprovecharon la experiencia profesional de los participantes y percibían rápidamente los perfiles de los profesionales que conformaban los grupos. Lo anterior les permitía adaptar con flexibilidad el contenido de la sesión al contexto de los participantes sin necesidad de forzar la narrativa del caso a la realidad de la empresa. Estas dinámicas de colaboración permitieron comunicar realidades complejas para que fueran entendidas con fluidez por los participantes. Las adaptaciones de la narrativa se dieron en áreas más específicas -ej., mercadeo, finanzas-, no tanto en otras áreas más genéricas -ej., factor humano-. De hecho, la formación de habilidades directivas se impartía de modo similar en todos los PCM, indistintamente del entorno empresarial. 
Uno de los efectos de todos los PCM, especialmente los exitosos, es que promovían el networking, el cual era posible gracias a la interacción con perfiles similares de la misma empresa que, de otra manera, no se habrían relacionado cara a cara -ej. grandes corporaciones-. A diferencia de los programas abiertos, donde se conocen a personas de sectores y empresas diferentes, en los PCM la cercanía que propiciaron las sesiones generó un ambiente colaborativo para promover el networking de una misma organización. El networking se facilitaba cuando la empresa trazaba objetivos de aprendizaje y de comunicación definiendo tendencias internas de acción estratégica. De este modo, los PCM también generaron una visión más colaborativa de la organización y facilitaron la toma de decisiones en función con la estrategia corporativa.

Las empresas asumían que la escuela de negocios haría una implementación impecable del PCM. Por ello, las escuelas de negocios debían gestionar los imprevistos de manera que la empresa no percibiera los inconvenientes graves. Si se transgredía esa imperceptibilidad del cliente y los problemas se salían de control, los PCM dejaban de ser exitosos y se convertían en PCM con dificultades. No bastaba tener control sobre unas pocas variables de éxito, era necesario que todas calzaran entre ellas. Esto se debía a que la colaboración dentro de los PCM funcionaba como un sistema integrado, donde hay subsistemas que deben encajar con otros para un desempeño óptimo -ej. perfil de participantes, escogencia de profesores y casos-.

Uno de los factores que distinguió a los PCM exitosos de los no exitosos era su manejo de la retroalimentación. Tanto las valoraciones cuantitativas -ej. resultados de las encuestas de satisfacción- como las cualitativas -ej. comentarios de pasillo- ayudaron a articular numerosas actividades de colaboración. En algunas ocasiones, estas actividades de retroalimentación fueron tan poderosas que a veces recrearon las actividades de diseño para ceder incluso parcialmente el control a los participantes del PCM. Con frecuencia, se debían hacer cambios rápidos mientras se llevaba a cabo el PCM. Por ejemplo, el profesorado que intervino en PCM exitosos era versátil: fue capaz de recibir retroalimentación de inmediato y hacer ajustes con prontitud a las recomendaciones que recibió -ej., énfasis en un tema, sustituir un caso para la siguiente sesión-. Los PCM menos exitosos no presentaban tanta rapidez ni capacidad de respuesta ante tales imprevistos.

\subsection{Discusión}


El propósito de este artículo es ofrecer una exploración de los PCM en América Latina, basados en un estudio comparativo de casos. Las dinámicas entre los agentes que intervienen a lo largo del desarrollo del un PCM sugieren que existen tres dimensiones críticas de la colaboración: diagnóstico, diseño e implementación.

Los hallazgos evidencian que las habilidades directivas de ambas organizaciones en el diagnóstico, diseño e implementación de los PCM es decisivo para su desarrollo eficiente (Mattessich y Johnson, 2018). En la dimensión de diagnóstico, las personas encargadas de AyD y líderes organizacionales contribuyen con su creatividad en la resolución de problemas de la empresa proporcionando información sobre la organización. Posteriormente, en la fase de diseño, los clientes coadyuvan a definir los perfiles de los potenciales participantes y contenidos del PCM. En la fase de implementación, los clientes ayudan a garantizar la calidad a través de evaluaciones y comentarios y brindan asistencia al personal durante las actividades de implementación. De hecho, se encontró que el profesorado comparte su experiencia profesional y académica- con los participantes de los PCM a través del análisis de casos: utilizan las problemáticas de los casos para compartir conocimiento teórico y relevante para las situaciones que atraviesa la empresa (Anderson et al., 2014).

Además, los grupos homogéneos forman PCM eficientes en edad, profesión, potencial de desarrollo y actitud hacia el aprendizaje para lograr un diseño variado y multidisciplinario (Saldana, 2016). La composición de las personas participantes en un grupo se construye mediante una actividad de "curaduría", producida por dinámicas de colaboración entre empresas y escuelas de negocios. Mientras que los grupos homogéneos permiten el aprendizaje y las interacciones de las personas asistentes, los grupos heterogéneos limitan el conocimiento y socavan la eficiencia de los PCM. Si los problemas organizacionales que resuelven los PCM son particulares -a diferencia de los programas abiertos-, las soluciones también deben acotarse, incluso en la elección de las personas participantes para superar los límites del conocimiento (Ambrose et al., 2012).

Las dinámicas de colaboración de los PCM en varios países de América Latina tienen patrones similares (Szkudlarek et al., 2013). Se identificó que las dinámicas de colaboración ocurren en paralelo y en un orden variado: no es un proceso que avanza linealmente. La capacidad de los PCM para perfeccionarse mediante la retroalimentación y las actividades de iteración también ayuda a superar las dificultades. Proveedores y clientes interactúan dentro de los límites de colaboración para proporcionar recursos que actualizan las necesidades y resuelven problemas. De acuerdo con la información analizada en este estudio, las escuelas 
de negocios que brindan oportunidades de colaboración más significativas a los clientes tienen mayores posibilidades de éxito en los PCM y viceversa. De igual forma, cuanto mayor es la calidad de las interacciones de los clientes en el diseño de los cursos, contenidos e implementación de las sesiones, mayor es la eficiencia en el desempeño del PCM.

En las dinámicas de colaboración para desarrollar los PCM, el profesorado de la escuela de negocios y las personas de la empresa desarrollan un entendimiento común en torno al ámbito de los negocios (Kwan, 2019). Así, el lenguaje de los negocios les confiere suficiente cercanía como para compartir proyectos comunes, al tiempo que divergen en otras actividades propias de su gestión. La existencia de estas divergencias hace necesario habilitar un espacio colaborativo en el cual se desarrollen las concepciones, modos y herramientas de intercambio adecuados para desarrollar el producto o servicio deseado. Este estudio permite afirmar que los mecanismos mediante los cuales las empresas y escuelas de negocios desarrollan los PCM de manera exitosa son debidos a dinámicas eficientes de colaboración (Mattessich y Johnson, 2018).

La dinámica de retroalimentación optimiza la entrega del PCM, mejora las sucesivas ediciones de otros programas cuando hay recompras e incluso mejora los PCM cuando tienen módulos sucesivos. La retroalimentación durante la implementación de los PCM exitosos permite optimizar los programas. Esto sucede gracias a que los responsables de PCM y AyD interactúan y colaboran más allá de las fronteras establecidas por una relación contractual, con el propósito de tener un mayor control sobre la experiencia de los agentes. Los comentarios de los participantes, los gerentes de AyD y los líderes de la organización permiten la colaboración in-situ para la mejora de los PCM. De esta manera se expanden los límites de la colaboración entre la empresa y la escuela de negocios (Dover et al., 2018).

\section{Conclusiones}

Las escuelas de negocios a nivel mundial ofrecen productos y servicios relativamente estandarizados: maestrías, programas ejecutivos y PCM. A pesar de ser estos productos tradicionales, todos ellos sufren modificaciones a lo largo del tiempo, y los PCM no son la excepción. Por tanto, las empresas deben dar especial énfasis al estudio de los PCM, no solo por la generación de ingresos que suponen, sino porque hay numerosos competidores y formatos -ej., plataformas en línea-, que exigen entender y reinventar el formato tradicional de los PCM. En este contexto, las dinámicas de colaboración pueden potenciar su desarrollo competitivo. 
Las empresas esperan que sus participantes tengan un mejor desempeño profesional luego de cursar el PCM, aún y cuando no hayan definido estándares de medición, o ni siquiera hayan manifestado esta necesidad explícitamente. Para lograr una buena medición del desempeño de los PCM, deben construirse indicadores conjuntos -mediante dinámicas de colaboración-, entre las empresas y las escuelas de negocios. Los PCM deben desarrollar habilidades para la toma de decisiones y esas mejoras en el desempeño individual y organizacional deben surtir un efecto en el mediano o corto plazo, no solo en el largo plazo.

Como limitante a la investigación está que las escuelas de negocios participantes forman parte de una red de instituciones hermanas, las cuales se encuentran en Latinoamérica y usan, como estrategia preponderante de enseñanza, el método del caso. Considerando que este fue un estudio exploratorio emprendido antes del inicio de la pandemia COVID-19, investigaciones futuras podrían validar estos hallazgos en otras disciplinas distintas de la administración de empresas, así como en otros formatos no presenciales diferentes al método del caso: educación virtual e híbrida, por ejemplo.

La literatura de los negocios ha estudiado poco los PCM. Las relaciones de colaboración entre empresas y escuelas de negocios resultan esenciales en los procesos de vinculación entre las instituciones de educación superior y el sector productivo. Comprender los factores que hacen más eficientes estos procesos de transmisión del conocimiento puede ser un punto de partida para futuras investigaciones, diseño y rediseño organizacional de las unidades de PCM para que logren fortalecer las escuelas de negocios y otras unidades académicas en la promoción de su autonomía y sostenibilidad.

A pesar de que esta es una investigación que involucra varios países, no se efectúa una aproximación trans-cultural. En próximas investigaciones es posible aplicar este enfoque para analizar y validar las variables encontradas a lo largo de diversos contextos geográficos y de negocios, más allá de Latinoamérica, también bajo el enfoque de la colaboración. Otros temas tales como los nuevos métodos de enseñanza y aprendizaje, sistemas de medición de los PCM pueden ser sujeto de estudios futuros bajo el mismo enfoque colaborativo. 


\section{Referencias}

Ambrose, Susan., Bridges, Michael., DiPietro, Michele., Lovett, Marsha., y Norman, Marie. (2012). How learning works: seven research-based principles for smart teaching. California: Jossey-Bass.

Anderson, Espen., Schiano, William Thomas., y Schiano, Bill. (2014). Teaching with cases: A practical guide. Harvard Business Press.

Asplund, Rita. (2004). The provision and effects of company training: A brief review of literature. ETLA Discussion Papers, The Research Institute of the Finnish Economy (ETLA), (907), $1-24$.

Barnett, Kent., y Mattox, John. (2010). Measuring success and ROI in corporate training. Journal of Asynchronous Learning Network, 14(2), 28-44.

Boyce, Carolyn., y Neale, Palena. (2006). Conducting In-Depth Interviews: A Guide for designing and conducting in-depth interviews for Evaluation Input. Pathfinder International Tool Series: Monitoring and Evaluation - $2 . \quad$ Retrieved from http://www2.pathfinder.org/site/DocServer/m e tool series indepth interviews.pdf?docl $\underline{D}=6301$

Büchel, Bettina., y Antunes, Don. (2007). Reflections on executive education: The user and provider's perspectives. Academy of Management Learning and Education, 6(3), 401-411.

Carrigtoncrisp. (2017). The Impact of Executive Education Report: A review of current practice \& trends. London.

Doh, Jonathan., y Stumpf, Stephen A. (2007). Interview and Commentary: Executive Education: A View from the Top. Academy of Management Learning \& Education, 6(3), 388-400.

Dover, Philip A., Manwani, Sharm., y Munn, David. (2018). Creating learning solutions for executive education programs. International Journal of Management Education, 16(1), 80 91. https://doi.org/10.1016/j.ijme.2017.12.002

Doz, Yves. (2007). The evolution of cooperation in strategic alliances: Initial conditions or learning processes? Strategic Management Journal, 17(1), 55-83. Doi https://doi.org/10.1002/smj.4250171006

Eisenhardt, Kathleen. (1989). Building Theories from Case Study Research. Academy of Management Review, 14(4), 532-550.

Garavan, Thomas., McCarthy, Alma., Lai, Yanqing., Murphy, Kevin., Sheehan, Maura., y Carbery, Ronan. (2021). Training and organisational performance: A meta-analysis of temporal, institutional and organisational context moderators. Human Resource Management Journal, 31(1), 93-119. https://doi.org/10.1111/1748-8583.12284

Garavan, Thomas., McCarthy, Alma., Sheehan, Maura., Lai, Yanqing., Saunders, Mark., Clarke, Nicholas ... Shanahan, Valerie. (2019). Measuring the organizational impact of training: The need for greater methodological rigor. Human Resource Development Quarterly, 30(3), 291-309. https://doi.org/10.1002/hrdq.21345 
Glaser, Barney G., y Strauss, Anselm L. (1999). The Discovery of Grounded Strategies for Qualitative Research. Aldine. New York: Routledge.

Goossen, Martin C. (2015). Interorganizational Collaboration, Intraorganizational Networks, and Firm Innovation. Academy of Management Proceedings, 2015(1), 10595-10595. https://doi.org/10.5465/ambpp.2015.43

Gulati, Ranjay., Wohlgezogen, Franz., y Zhelyazkov, Pavel. (2012). The Two Facets of Collaboration: Cooperation and Coordination in Strategic Alliances. Academy of Management Annals, 6(1), 531-583.

Harrison, Michael., y Shirom, Arie. (1999). Organizational Diagnosis and Assessment: Bridging Theory and Practice. Thousand Oaks, California. http://sk.sagepub.com/books/organizational-diagnosis-and-assessment

Haskins, Mark., Centini, Lou., y Shaffer, George (2017). Ideas for growing executive education revenue: codification and catalyst. Journal of Management Development, 36(4), 581-597.

Kets De Vries, Manfred., y Korotov, Konstantin. (2007). Creating transformational executive education programs. Academy of Management Learning and Education, 6(3), 375-387. https://doi.org/10.5465/amle.2007.26361627

Kirkpatrick, Donald., y Kirkpatrick, James. (2007). Implementing The Four Levels. Springer Series in Optical Sciences. California: Berrett-Koehler Publishers.

Knoben, Joris., y Oerlemans, Leon. (2006). Proximity and inter-organizational collaboration: A literature review. International Journal of Management Reviews, 8(2), 71-89. https://doi.org/10.1111/j.1468-2370.2006.0012.x

Kwan, Lisa. (2019). The Collaboration Blind Spot. Harvard Business Review. https://hbr.org/2019/03/the-collaboration-blind-spot

Lai, Emily R. (2011). Collaboration: A Literature Review. Research Reports. Pearson.

Leach, William., Weible, Christopher., Vince, Scott., Siddiki, Saba., and Calanni, John. (2014). Fostering learning through collaboration: Knowledge acquisition and belief change in marine aquaculture partnerships. Journal of Public Administration Research and Theory, 24(3), 591-622.

Lockhart, James. (2013). Executive Education: Can it Be Too Good? Journal of Executive Education, 12(1).

Madsen, Peter., y Desai, Vinit. (2010). Failing to Learn? The effects of failure and success on organizational learning in the global orbital launch vehicule industry. The Academy of Management Journal, 53(3), 451-476.

Mattessich, Paul., y Johnson, Kirsten. (2018). Collaboration: What Makes It Work. A Review of Research Literature on Factors Influencing Successful Collaboration (3rd ed.). New York, N.Y: Fieldstone Alliance. 
Merriam, Sharan. (1998). Case study research in education: A qualitative approach (2nd ed.). San Francisco: Jossey-Bass Publishers.

Montgomery, Laura. (2016). Choosing your executive-education programme: custom vs. openenrolment. $\quad$ https://execed.economist.com/career-advice/choosing-yourprogramme/choosing-your-executive-education-programme-custom-vs-open

Murray, John. (2014). Designing and Implementing Effective Professional Learning. Thousand Oaks, California: Corwin.

Myrsiades, Linda. (2001). Looking to lead: A case in designing executive education from the inside. Journal of Management Development, 20(9), 795-812.

Pera, Rebecca., Occhiocupo, Nicoletta., y Clarke, Jackie. (2016). Motives and resources for value co-creation in a multi-stakeholder ecosystem: A managerial perspective. Journal of Business Research, 69(10). https://doi.org/10.1016/j.jbusres.2016.03.047

Saldana, Johnny. (2016). The Coding Manual for Qualitative Researchers. The coding manual for qualitative researchers (3rd ed.). London: SAGE Publications Ltd.

Senge, Peter. (1990). The fifth discipline: the art and practice of the learning organization. New York: Doubleday-Currency.

Strebel, Paul. (2007). Mastering Executive Education: How to Combine Content with Context and Emotion: The IMD Guide. Academy of Management Learning \& Education, 6(3), 423-425.

Szkudlarek, Betina., Mcnett, Jeanne., Romani, Laurence., y Lane, Henry. (2013). The past, present, and future of cross-cultural management education: The educators' perspective. Academy of Management Learning and Education, 12(3), 477-493. http://dx.doi.org/10.5465/amle.2012.0233.

Yin, Robert. (2008). The Case Study Crisis: Some Answers. Administrative Science Quarterly, 26(1), 58-65.

Yin, Robert. (2009). How to Do Better Study Cases. In L. Bickman and D. J. Rog (Eds.), The SAGE Handbook of Applied Social Research Methods (2nd ed., pp. 254-283). SAGE Publications Inc.

Yin, Robert. (2014). Case Study Research: Design and Methods (5th ed.). California: SAGE Publications, Inc. 


\section{Anexos}

\subsection{Anexo 1: PCM estudiados}

Tabla 1

Perfil de las empresas participantes

(Argentina, Brasil, Colombia, Ecuador, México y Perú, 2017-2018)

\begin{tabular}{|c|c|c|c|c|c|}
\hline $\begin{array}{l}\text { Nombre } \\
\text { (hipotético) }\end{array}$ & $\begin{array}{l}\text { Sector de la } \\
\text { empresa }\end{array}$ & $\begin{array}{l}\text { Cantidad de } \\
\text { colaboradore } \\
\text { s }\end{array}$ & $\begin{array}{l}\text { País donde } \\
\text { se impartió }\end{array}$ & $\begin{array}{l}\text { Perfil de } \\
\text { participantes }\end{array}$ & $\begin{array}{l}\text { Tipo de } \\
\text { programa }\end{array}$ \\
\hline PCM \#1 & Petrolero & $\begin{array}{l}\text { 1.100+ } \\
\text { (en la región) }\end{array}$ & Argentina & $\begin{array}{l}\text { Mandos } \\
\text { medios }\end{array}$ & $\begin{array}{l}\text { Liderazgo } \\
\text { gerencial }\end{array}$ \\
\hline PCM \#2 & Financiero & $1.300+$ & Ecuador & Gerentes & $\begin{array}{l}\text { Habilidades } \\
\text { directivas }\end{array}$ \\
\hline PCM \#3 & Financiero & $1.500+$ & Ecuador & Gerentes & $\begin{array}{l}\text { Gerencia } \\
\text { financiera }\end{array}$ \\
\hline PCM \#4 & Construcción & $1.500+$ & Colombia & Gerentes & $\begin{array}{l}\text { Habilidades } \\
\text { gerenciales }\end{array}$ \\
\hline PCM \#5 & Financiero & $40.000+$ & México & Gerentes & $\begin{array}{l}\text { Liderazgo } \\
\text { gerencial }\end{array}$ \\
\hline PCM \#6 & $\begin{array}{l}\text { Farmacéutic } \\
\text { o }\end{array}$ & $5.300+$ & México & Gerentes & $\begin{array}{l}\text { Liderazgo } \\
\text { gerencial }\end{array}$ \\
\hline PCM \#7 & $\begin{array}{l}\text { Educación } \\
\text { superior }\end{array}$ & $150+$ & México & $\begin{array}{l}\text { Consejos } \\
\text { directivos }\end{array}$ & $\begin{array}{l}\text { Juntas } \\
\text { directivas }\end{array}$ \\
\hline PCM \#8 & Financiero & $47.000+$ & Brasil & Gerentes & $\begin{array}{l}\text { Habilidades } \\
\text { gerenciales }\end{array}$ \\
\hline PCM \#9 & Salud & $700+$ & Perú & $\begin{array}{l}\text { Mandos } \\
\text { medios }\end{array}$ & $\begin{array}{l}\text { Habilidades } \\
\text { gerenciales }\end{array}$ \\
\hline
\end{tabular}

Fuente: Elaboración propia, 2021

Tabla 2

Composición de las entrevistas en los casos de estudio (Argentina, Brasil, Colombia, Ecuador, México y Perú, 2017-2018)

\begin{tabular}{lcclccc}
\hline $\begin{array}{l}\text { Casos de } \\
\text { éxito (5) }\end{array}$ & $\begin{array}{c}\text { Entrevistas } \\
\text { a la empresa }\end{array}$ & $\begin{array}{c}\text { Entrevistas } \\
\text { a la escuela }\end{array}$ & $\begin{array}{l}\text { Casos } \\
\text { dificultades (4) }\end{array}$ & $\begin{array}{c}\text { Entrevistas } \\
\text { la empresa }\end{array}$ & $\begin{array}{c}\text { Entrevistas } \\
\text { a la escuela }\end{array}$ \\
\hline PCM \#2 & 3 & 3 & PCM \#1 & 1 & 5 \\
PCM \#3 & 3 & 5 & PCM \#4 & 3 & 4 \\
PCM \#5 & 3 & 4 & PCM \#7 & 4 & 4 \\
PCM \#6 & 3 & 5 & PCM \#9 & 3 & 3 \\
PCM \#8 & 1 & 5 & & & \\
\hline
\end{tabular}

Fuente: Elaboración propia, 2021 


\subsection{Anexo 2: Guía de entrevista semi estructurada}

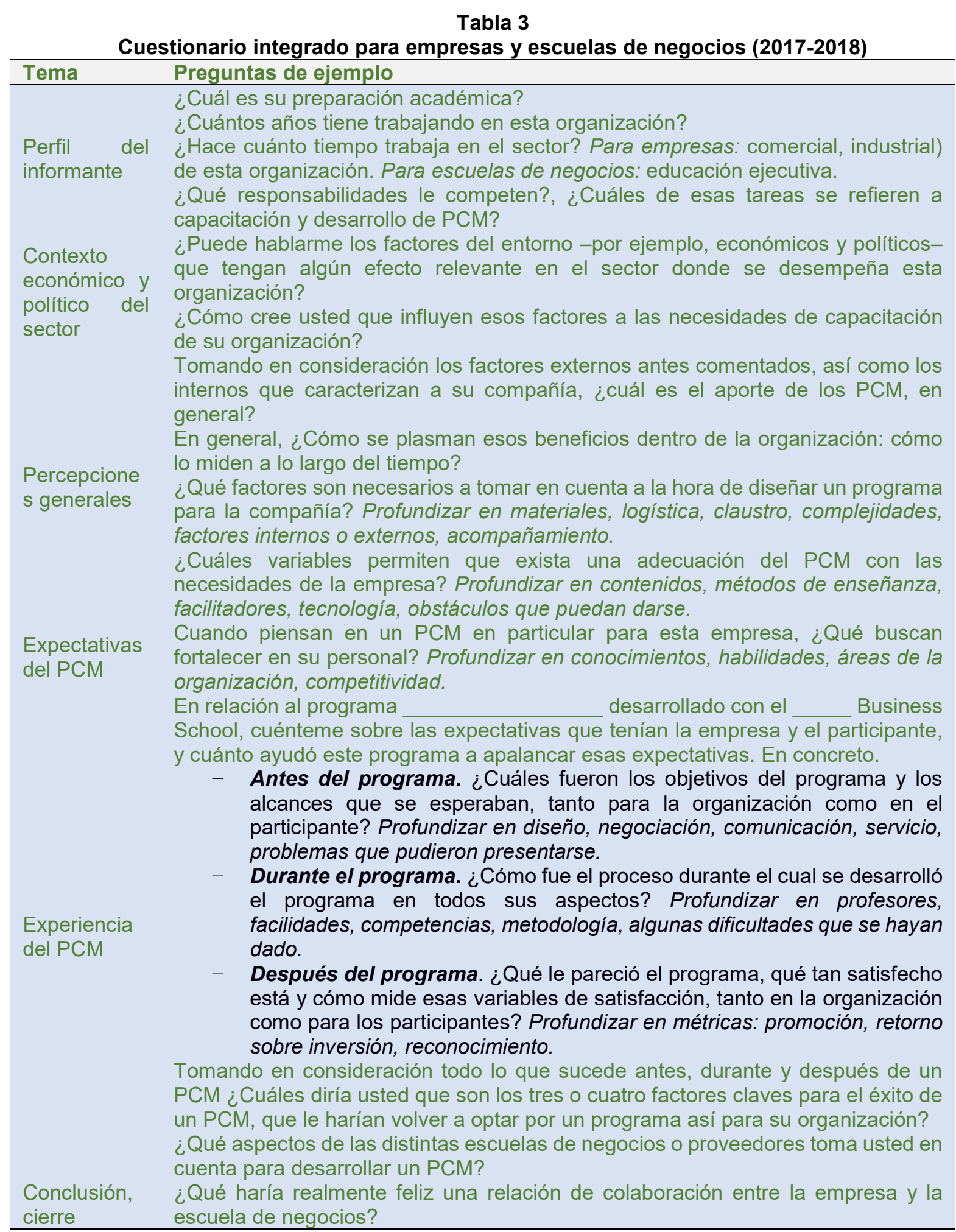

Fuente: Elaboración propia, 2021 


\subsection{Anexo 3:}

Tabla 4. Información adicional para la fundamentación de variables

\section{Diagnóstico}

"Para entender al cliente, hay que tener contacto con él, entenderlo, y no darle lo que tenés de estándar. Entender al cliente y su necesidad, y después tener una flexibilidad en la capacidad de adaptar la solución a la realidad" (1:110).

"Es una venta consultiva que no la puede hacer un "junior" porque, generalmente, tus clientes, las personas con que trato de reunirme, son personas que conocen muy bien lo que necesitan o, si no lo conocen, necesitan tu asesoría, entonces es más una venta consultiva" (2:29).

"Ese es uno de los riesgos: que el diseño del programa responda a lo que gestión humana quiere, pero no necesariamente a lo que la empresa necesita" (4:36).

"Fueron varios meses de trabajo, tuvimos infinidad de reuniones, fuimos a visitarlos a sus diferentes locales, conversamos con diferentes directivos, ellos también vinieron, hubo una cercanía importante. Eso también se manifestó en la inauguración del programa y la clausura" (9:28).

\section{Diseño}

"Otro punto importante es cuidar el perfil de participante que va a asistir al programa. Si no, no resulta fructífero, y no es una buena inversión, ni de tiempo, ni en recursos financieros, para alguien que no lo va a aprovechar"(5:43).

"Debe haber también cierta homogeneidad en el nivel de los participantes; o sea, si tú mezclas gerentes con jefes, causa cierto desnivel, igual que en una maestría ejecutiva se pide que haya un cierto número de años de experiencia, una cierta formación" (3:102).

"Los profesores eran nacionales e internacionales, muy bien preparados. Algunos contaban con un doctorado de universidades importantes, y otros gozaban de amplia experiencia empresarial" (2:32).

"Nos pidieron a unos profesores internacionales. Acudimos un poco a las escuelas hermanas cercanas, porque si no, se complicaba un poco, y decidimos pedir una persona de Chile y una persona de Colombia que ya conocíamos. El porcentaje de escuelas hermanas nunca ha superado el $20 \%$. El $80 \%$ lo hacemos con profesores nacionales" (2:35).

\section{Implementación}

"Nosotros tuvimos veintidós grupos, yo creo, y en todas las evaluaciones nosotros fuimos calibrando los casos de acuerdo a las evaluaciones, de manera que se pudiera percibir el paralelo, que las personas pudieran llegar al objetivo: el aprendizaje. Incluso tuvimos que cambiar algunos casos, para poder llegar a eso, y fue un trabajo de acompañamiento muy cercano el que tuvimos que hacer para lograr eso" (8:36).

"El programa se basó principalmente en el análisis de casos para la toma de decisiones. Se trataba de historias reales que se dieron en el mundo de los negocios, desde empresas muy importantes, hasta otras no tan relevantes, pero que constituían ejemplos interesantes para que los profesores pudieran entregar una enseñanza particular. El estudio de los casos se hacía de manera individual; posteriormente, se analizaban en un foro grupal y, finalmente, se discutía en una sesión plenaria donde intervenian todos los participantes" (2:31).

"Cuando van terminando el programa, vamos haciendo un acompañamiento con ellos para ver cómo van mejorando en los temas de liderazgo (...). Esto les ayuda a complementar los temas que necesitan para seguir creciendo en el banco" (5:57).

"Lo que yo puedo apreciar de un programa in-company es que te permite que el directivo tenga una visión holística, si lo puedo llamar así, en cuanto a todos los factores que influyen en la empresa" (5:29).

Fuente: Elaboración propia, 2021 


\subsection{Anexo 4:}

Figura 3. Libro de códigos (vista parcial)

Primer ciclo de codificación Categorías

\begin{tabular}{|c|c|c|}
\hline Colaboración & $\begin{array}{c}\text { Colaboración dentro de } \\
\text { las organizaciones }\end{array}$ \\
\cline { 2 - 3 } & $\begin{array}{c}\text { Colaboración dentro de } \\
\text { la escuela de negocios }\end{array}$ \\
\hline $\begin{array}{c}\text { Colaboración entre la } \\
\text { empresa y la escuela de } \\
\text { negocios }\end{array}$ \\
\hline
\end{tabular}

\begin{tabular}{|c|c|c|}
\hline Diseño & $\begin{array}{c}\text { Diseño del CEPP y } \\
\text { adaptación }\end{array}$ & Diseño \\
\hline
\end{tabular}

\begin{tabular}{|c|c|}
\hline Experiencia & $\begin{array}{c}\text { Experiencia antes } \\
\text { del PCM }\end{array}$ \\
\cline { 2 - 2 } & $\begin{array}{c}\text { Experiencia durante } \\
\text { el PCM }\end{array}$ \\
\hline \hline $\begin{array}{c}\text { Experiencia después } \\
\text { del PCM }\end{array}$ \\
\hline
\end{tabular}

\begin{tabular}{|c|c|}
\hline Evaluación & $\begin{array}{c}\text { Evaluación de la } \\
\text { empresa }\end{array}$ \\
\cline { 2 - 2 } & $\begin{array}{c}\text { Evaluación de la } \\
\text { escuela de negocios }\end{array}$ \\
\hline $\begin{array}{c}\text { Evaluación entre } \\
\text { empresa y escuela de } \\
\text { negocios }\end{array}$ \\
\hline $\begin{array}{c}\text { Percepción: prestigio de } \\
\text { la escuela de negocios }\end{array}$ \\
\hline
\end{tabular}

Fuente: Elaboración propia, 2021

\section{Segundo ciclo de codificación (axial)}

Códigos

\section{Dinámicas}

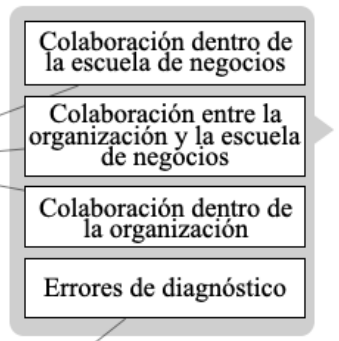

1. Diagnóstico

\section{Diseño}

Diseño de los

Comprensión de las necesidades del cliente

\begin{tabular}{|c|}
\hline Extensión del PCM \\
\hline $\begin{array}{c}\text { Homogeneidad del } \\
\text { grupo de participantes }\end{array}$ \\
\hline \hline $\begin{array}{c}\text { Pertinencia del diseño } \\
\text { del PCM }\end{array}$ \\
\hline $\begin{array}{c}\text { Rentabilidad y valor del } \\
\text { PCM }\end{array}$ \\
\hline
\end{tabular}

Cuidado y atención de
participantes del PCM

Evaluacion en general

\section{Implementación}

Evaluación en general

Medidas de
contingencia

che

3. Implementación

"logística" del PCM 
Revista indizada en

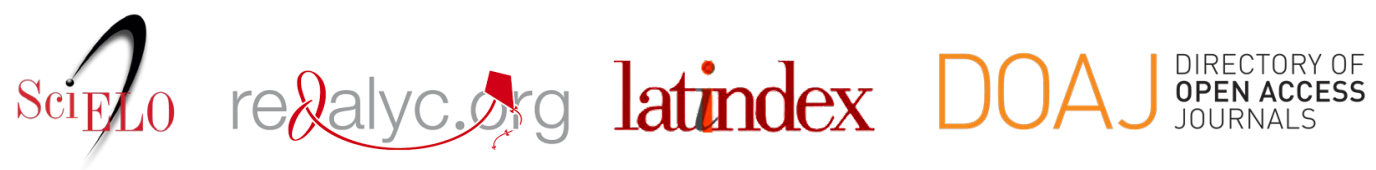

Distribuida en las bases de datos:

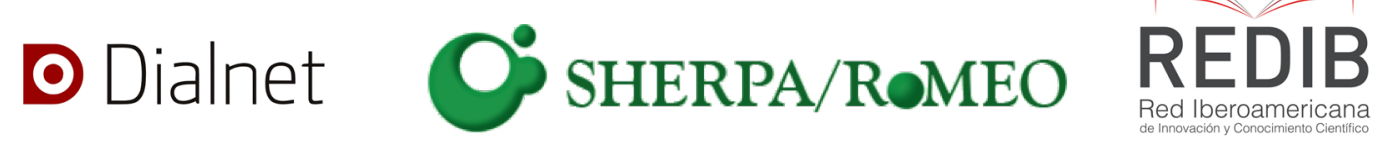

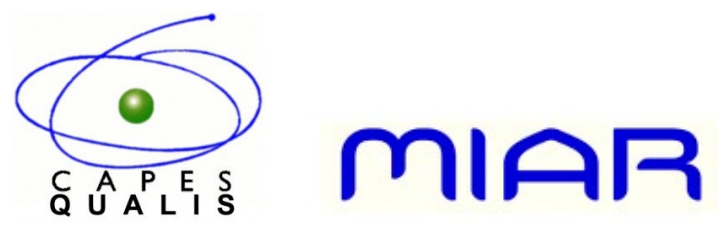

